every 4 weeks, with the primary endpoints assessed at Week 52 , in patients with moderate to severe SLE despite standardof-care treatment. ${ }^{1,2}$ BICLA responses were compared between anifrolumab $300 \mathrm{mg}$ and placebo groups, and robustness of BICLA responses was assessed across protocol-defined subgroups.

Results TULIP-2 (anifrolumab, $\mathrm{n}=180$; placebo, $\mathrm{n}=182$ ) and TULIP-1 (anifrolumab, $\mathrm{n}=180$; placebo, $\mathrm{n}=184$ ) had comparable BICLA responses (figure 1). Across multiple subgroups, higher percentages of patients achieved BICLA responses at Week 52 in the anifrolumab vs placebo arms. There was concordance of BICLA responses favoring anifrolumab across the protocol-defined subgroups of baseline disease severity (SLEDAI-2K $<10$ points [difference 15.3\%, TULIP-2; 16.9\%, TULIP-1] vs $\geq 10$ points [difference $16.7 \%$, TULIP-2; $17.1 \%$, TULIP-1]) and baseline oral corticosteroid use (prednisone or equivalent $<10 \mathrm{mg} / \mathrm{d}$ [difference 20.1\%, TULIP-2; 16.2\%, TULIP-1] vs $\geq 10 \mathrm{mg} / \mathrm{d}$ [difference $12.0 \%$, TULIP-2; $17.7 \%$, TULIP-1]). Other subgroups including age, sex, age at onset, race, and anti-drug antibody status showed similar uniformity of response.

Conclusions The uniformity of robust BICLA response rates across prespecified subgroups in both phase 3 trials shows consistent clinical benefit of anifrolumab irrespective of patient baseline characteristics.

\section{INCIDENCE AND PREDICTORS OF ATHEROSCLEROTIC VASCULAR EVENTS IN A MULTICENTRE INCEPTION SLE COHORT}

Murray Urowitz, Dafna D Gladman, Jiandong Su, Vern T Farewell, Systemic Lupus International Collaborating Clinics (SLICC) Group. University of Toronto, Toronto, Canada

\subsection{6/lupus-2020-eurolupus.37}

Background/Purpose The prevalence of atherosclerotic vascular events (AVE) in published literature of an inception cohort with SLE is $10 \%$. We aimed to investigate the accrual and the associated factors of AVE in a multinational multiethnic inception cohort of patients with SLE.

Methods A large 33-centre multinational inception cohort of SLE patients was followed yearly according to a standardized protocol between 1999-2017. AVEs are attributed to atherosclerosis on the basis of SLE being inactive at the time of the event, and the presence of typical atherosclerotic changes on imaging or pathology and/or evidence of atherosclerosis elsewhere. Analysis included descriptive statistics, rate of AVE's per 1000 patient-years and univariable and multivariable relative risk regression models.

Results Of the 1848 patients enrolled, 1710 that had at least one follow up visit after enrolment comprised of the study sample. $88.6 \%$ were female, $49.4 \%$ Caucasian, $16.4 \%$ Black, $15.0 \%$ Asian, $15.5 \%$ Hispanic and $3.7 \%$ other. Disease duration at enrolment was $5.7 \pm 4.2$ months, mean age at enrolment was $34.7 \pm 13.4$ years and SLEDAI-2K was $5.4 \pm 5.4$. The prevalence of AVEs was $3.6 \%$ and the rate per 1000patient years was 4.6. Sixty-one patients had atherosclerotic events after the enrolment; their detailed events and numbers are listed in table 1.

Two multivariable models including the predictors with significant effects in the single factor analyses, one without the aCL/LA variable and one with this variable are presented in
Abstract 026 Table 1 Multivariate Models. Outcome: first AVE

\begin{tabular}{lllll}
\hline & \multicolumn{2}{l}{ Model (a) $[60 \text { events }]^{*}$} & \multicolumn{2}{l}{ Model $(b)[53 \text { events }]^{*}$} \\
\hline Predictor & HR $(95 \% \mathrm{Cl})$ & $\mathrm{p}$-value & HR $(95 \% \mathrm{Cl})$ & $\mathrm{p}$-value \\
\hline Prior other VEs & $4.00(1.55,10.3)$ & 0.004 & $4.76(1.80,12.60)$ & 0.002 \\
Female & $0.60(0.32,1.24)$ & 0.12 & $0.57(0.28,1.13)$ & 0.11 \\
Never vs ever smoked & $0.51(0.24,1.06)$ & 0.07 & $0.48(0.23,1.03)$ & 0.06 \\
Ex-smoker vs current & $0.67(0.30,1.48)$ & 0.33 & $0.53(0.23,1.21)$ & 0.13 \\
smoker & & & & \\
Anti-malarials & $0.54(0.32,0.91)$ & 0.02 & $0.52(0.29,0.92)$ & 0.02 \\
BMI 30-40 & $0.95(0.48,1.88)$ & 0.89 & $0.88(0.42,1.87)$ & 0.74 \\
BMI $\geq 40$ & $2.74(1.04,1.18)$ & 0.04 & $3.10(1.17,8.23)$ & 0.02 \\
aCL/LA ever & & & $1.73(0.95,3.12)$ & 0.07 \\
\hline
\end{tabular}

^ Missing values in the predictors led to fewer events in some analyses. BMI - body mass index; aCL/LA - anticardiolipin/lupus anticoagulant; VEs - vascular events

table 1. The inclusion of aCL/LA led to the exclusion of 405 patients. Prior other nonatherosclerotic vascular events and high BMI were predictive of first AVE while only antimalarial therapy demonstrated a highly significant protective effect, [HR (95\%CI): $0.54(0.32,0.91)]$, after adjustment for the other factors in the model.

Conclusion More effective control of classic atherosclerotic risk factors and more frequent use of antimalarial may have both contributed to controlling AVEs in this inception cohort.

\section{DEVELOPMENT AND VALIDATION OF A MULTIVARIABLE MODEL FOR 5-YEAR SURVIVAL IN SYSTEMIC LUPUS ERYTHEMATOSUS-ASSOCIATED PULMONARY ARTERIAL HYPERTENSION: CSTAR-PAH COHORT STUDY}

Jingge Qu, Junyan Qian, Jiuliang Zhao, Qian Wang, Mengtao Li, Xiaofeng Zeng on behalf of CSTAR-PAH collaborators. Dept. of Rheumatology, Peking Union Medical College Hospital, Peking Union Medical College and Chinese Academy of Medical Sciences, National Clinical Research Center for Immunologic Diseases, Ministry of Science and Technology, Key Laboratory of Rheumatology and Clinical Immunology, Ministry of Education, Beijing, China

\subsection{6/lupus-2020-eurolupus.38}

Background Pulmonary arterial hypertension (PAH) is the major mode of death in systemic lupus erythematosus (SLE), but there is no validated algorithm to identify those at highest risk.

Methods A prognostic model was developed from a multicenter, longitudinal cohort study of 310 consecutively evaluated patients with SLE-associated PAH. The study was conducted from November 2006 to January2019. Death was the primary outcome. The model was developed using Cox proportional hazards regression modeling. We developed a prognostic index (PI), summing the number of risk points corresponding to weighted covariates, which were used to configure the nomogram. Internal validation of the nomogram was assessed by discrimination and calibration using bootstrapping.

Results Of the 310 patients included in the study, 68 deaths (22.2\%) occurred at a median follow-up of 4.9 (interquartile range [IQR] 3.2-6.3 years) years. The final prognostic model included 6 variables: $\mathrm{N}$ terminal-pro brain natriuretic peptide (NT-proBNP), Lactic Dehydrogenase (LDH), Direct Bilirubin 